\title{
Bilateral Vesicoureteric Reflux may be a Rare Association in Lesch-Nyhan Syndrome: A Case Report
}

\author{
HABIBUR RAHMAN ${ }^{1}$, MD. FIROZ ANJUM², SUSMITA BISWAS ${ }^{3}$, SYED SAIMUL HUQUE ${ }^{4}$, \\ AFROZA BEGUM ${ }^{5}$
}

\section{Introduction}

Lesch-Nyhan syndrome (LNS) was first described in 1964 as an X-linked recessive metabolic disorder, caused by the complete absence of hypoxanthine guanine phosphoribosyltransferase (HGPRT) activity. ${ }^{1}$ Incidence is 1 in 380,000 live births. ${ }^{2}$ Although LNS syndrome is an $\mathrm{X}$-linked recessive disorder, there have been a few reported cases involving female patients. ${ }^{3}$ The loss of enzyme function is caused by gene mutations on the long arm of the X-chromosome. ${ }^{4}$ This enzyme catalyses the reaction of hypoxanthine or guanine with phosphoribosyl pyrophosphateto form inosinic and guanylic acids. The increased production of purine leads to an accumulation of uric acid in the blood that leading to hyperuricemia. ${ }^{5}$ Children with LNS may develop all clinical signs of gout such as uricosuria, urinary tract calculi, tophaceous gouty arthritis and nephrolithiasis. Usually these patients die in the second or third decade of life from infection or renal failure. ${ }^{3,5-7}$ Therefore genetic counseling is essential for appropriate prevention. ${ }^{7}$ Prenatal diagnosis of LNS can be made by amniocentesis, $, 8,9$ hair root analysis ${ }^{5}$ or chorion biopsy ${ }^{6}$ and ultramicroscale enzymology. ${ }^{10}$

Affected males with this incurable disease are mentally and physically retarded and suffer from spasticity, choreoathetosis, hyperuricemia, and cerebral palsy. A consistent presentation in all cases of LNS is the abnormal compulsion toward selfmutilation usually reported after 1 year of age. ${ }^{4,11}$ Perioral self-mutilating behavior is thought to begin with the eruption of teeth; patients then begin to bite themselves. The behavior continues and results in partial or total destruction of perioral tissue especially the lower lip. Partial or

1. Professor, Department of Pediatric Nephrology, BSMMU, Dhaka.

2. MD Resident, Department of Pediatric Nephrology, BSMMU, Dhaka.

3. MD Resident, Department of Pediatric Nephrology, BSMMU, Dhaka.

4. Associate Professor, Department of Pediatric Nephrology, BSMMU, Dhaka.

5. Associate Professor, Department of Pediatric Nephrology, BSMMU, Dhaka.

Correspondence: Professor Dr. Md. Habibur Rahman, Chairman, Department of Pediatric Nephrology, BSMMU, Dhaka, Bangladesh, E-mail: mhrahman.bsmmu@gmail.com. complete amputation of fingers, toes, and tongue is also common. ${ }^{12}$ Major clinical manifestations occur usually in affected males and can be noticed first by 3 to 4 months of age. ${ }^{13}$ The first clinical symptoms are typically poor motor development, abnormal hypertonic muscle tone or spasticity, often leading to a misdiagnosis of cerebral palsy.

High serum uric acid level is usually the biochemical finding that prompts special testing for the specific diagnosis. However, infants with LNS may have borderline serum uric acid levels because of an increased renal clearance of uric acid. ${ }^{14}$ Diagnosis can be made by clinical features and confirmed by direct sequencing of all exons of the HPRT gene. ${ }^{15,16}$

Currently, there is no cure for the syndrome; prevention is therefore the standard of care. Over the years many inventive and effective approaches involving an assortment of hand restraints, dental mouth guards, and dental extractions have been utilized ${ }^{4,5,11}$ and with appropriate allopurinol treatment, renal function is generally preserved. ${ }^{17}$

LNS is usually associated with renal calculi and renal failure because of hyperuricemia and it has been established by several studies but vesicoureteric reflux as an association with LNS has not been reported till date so far.

So, the purpose of this case report is that vesicoureteric reflux (VUR)could be one of theassociations with the LNS besides the urinary tract calculi. The report provides an insight into the diagnosis and treatment of this condition in association with LNS.

\section{Case Report}

A 16 months old girl, $3^{\text {rd }}$ issue of consanguineous parents, hailing from chittagong, admitted with the complaints of dribbling of urine since birth, history of self biting of lips and tongue since 7 months of her age and fever for last 10 days. History revealed that child developed dribbling of urine since birth, and was diagnosed incorrectly as neurogenic bladder and was treated with catheterization. Since 7 months of age 
mother noticed of her daughter biting her own lips, fingers and tongue by her teeth. Sometimes cut injury over lips and fingers were associated and were not caused by any external stimuli. For last 10 days, she has been suffering from high grade fever, intermittent in nature and was associated with chills and rigor. She also had crying during micturition. The girl also had convulsion, generalized tonic clonic in nature, lasting for 4-5 minutes and relieved spontaneously without post aural symptoms. She was born by normal vaginal delivery at term at hospital with perinatal asphyxia. She has global developmental delayevidenced by neck control at 8 months of her age, she can sit but can not stand without support and unable to walk till date, she can speak only monosyllable, her ear and vision were intact. Nothing similar was noted in the family. She has history of taking oral valproic acid and resperidone for last 3 months for convulsion.

On examination: She was fretful, cut injury over upper and lower lips and also on the tip of the tongueand on both index fingers and thumbs (Fig.-1\&2). The girl was mildly pale, not edematous, vitals were within normal limit; Height 70cm (-2 SD), weight 8 kg (-2.6 SD), WHZ $25^{\text {th }}-50^{\text {th }}$ centile, OFC $42 \mathrm{~cm}$ (-3.6 SD).

Nervous system: General intellectual function was below average;hypotonea in lower limbs; sensory was intact; repetitive movement of left arm and left leg; gait yet not learnt to walk. Abdominal and genitourinary examination reveals normal finding. Immediate investigation that showed hemoglobin $9.6 \mathrm{gm} / \mathrm{dl}$, Erythrocyte sedimentation rate $44 \mathrm{~mm}$ in $1^{\mathrm{st}} \mathrm{hr}$, total count $14000 / \mathrm{cmm}$, neutrophil $41 \%$, lymphocyte $50 \%$, platelet $3700000 / \mathrm{cmm}$, red blood cell $4000 / \mathrm{cmm}$. Urine routine microscopic examination (RME): color hazy, pus cell 20-30/HPF. Urine culture and sensitivity showed no growth. Her serum uric acid level was 7 $\mathrm{mg} / \mathrm{dl}$. Serum electrolytes, serum creatinine, serum calcium, alkaline phosphate, serum phosphate, serum lactate, serum ammonia reveal normal findings. Ulrasonography (USG) reveals normal size kidney and post voidal residue $30 \mathrm{ml}$. MRI of brain reveals moderate cerebral atrophy (Fig-3). EEG reveals normal findings. Micturatingcystourethrography (MCU) reveals bilateral grade 2 VUR. X-ray of spine and KUB were normal. His 24 hours urinary uric acid level was 58 $\mathrm{mg} /$ day.On the basis of history, examination and investigations she was diagnosed as a case of LNS with Urinary tract infection (UTI) with VUR.But for confirmation of diagnosis of LNS, genetic testing is required to detect the mutation in HPRT1 gene but due to inadequate facility could not do this investigation.

During hospitalization she was treated with intravenous antibiotics, antipyretics, oral valproic acid and resperidone along with allopurinol. With above treatment she was improved by means of fever subsided, features of UTI improved and no seizure, self mutilating behavior decreased, so she was discharged with advice and follow up.

But after 7 days of discharge she developed swelling of the left leg which started from ankle and extended up to mid thigh. She was fretful, temperature $100^{\circ} \mathrm{F}$, radial pulse rate 110 per minutes, respiratory rate 24 breaths per minutes and blood pressure was 80/40 $\mathrm{mmHg}$ (Â50 ${ }^{\text {th }}$ centile), periphery was cool and capillary refill time was Â 3 seconds. Lower limb examination reveals swelling in right leg (from ankle joint to mid thigh). Skin was shiny, no color changes, temperature was normal compared to the opposite limb and was non tender, pulse in the lower limbs were normal. Investigation: complete blood count and urineRME revealed normal. Prothrombin time (PT) was 13.1 second, international normalized ratio(INR) 1.1; Ddimer $49.35 \mathrm{mg} / \mathrm{dl}$; fibrin degradation product (FDP) $10 \mathrm{ug} / \mathrm{dl}$. Color duplex of right lower limbs revealed thrombus in the right saphenous vein. So, she was diagnosed as LNSwith hypovolemic shock with deep vein thrombosis (right lower limb).

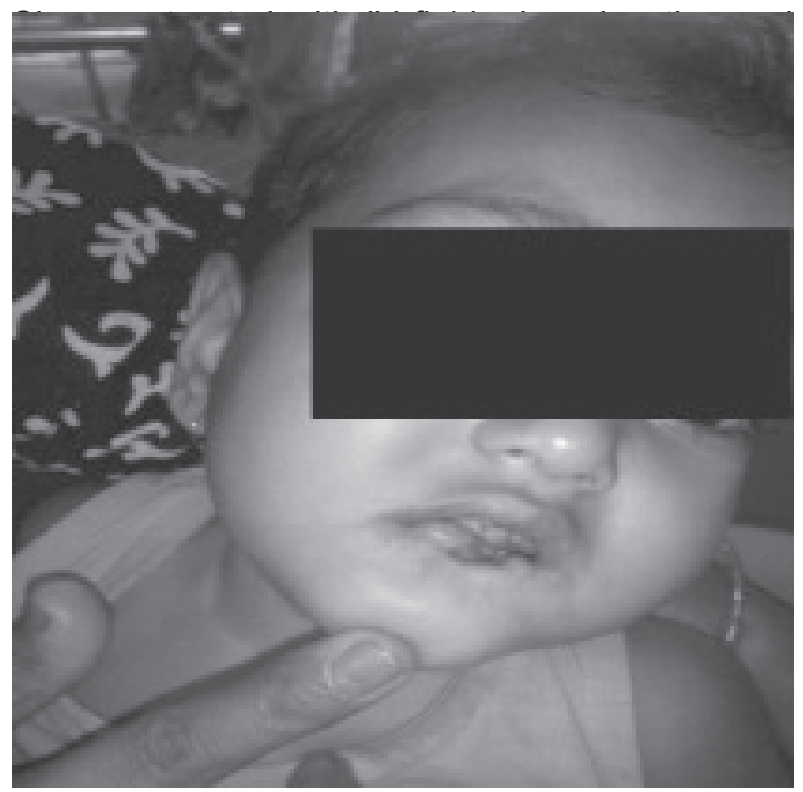

Fig-1: Cut injury over lower lip. 
subcutaneous low molecular weight heparin for 8 daysfollowed by oral warfarin for a period of 3-6 months and improved clinically and also by the means of laboratory (PT 49.2sec, INR 4.22). Patient was discharged with improvement and advised for follow up monthly with PT, INR, and color duplex. The aim of the warfarin therapy was to keep the INR within 2-3 for a period of 3-6 months and color duplex was advised to see whether thrombus resolved or not.

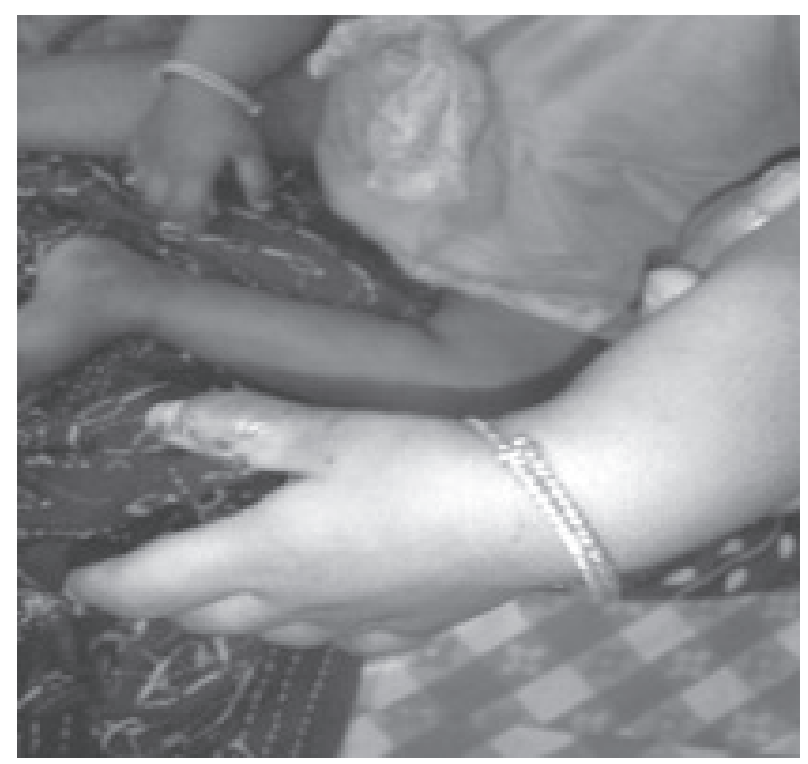

Fig-2: Cut injury over thumb.

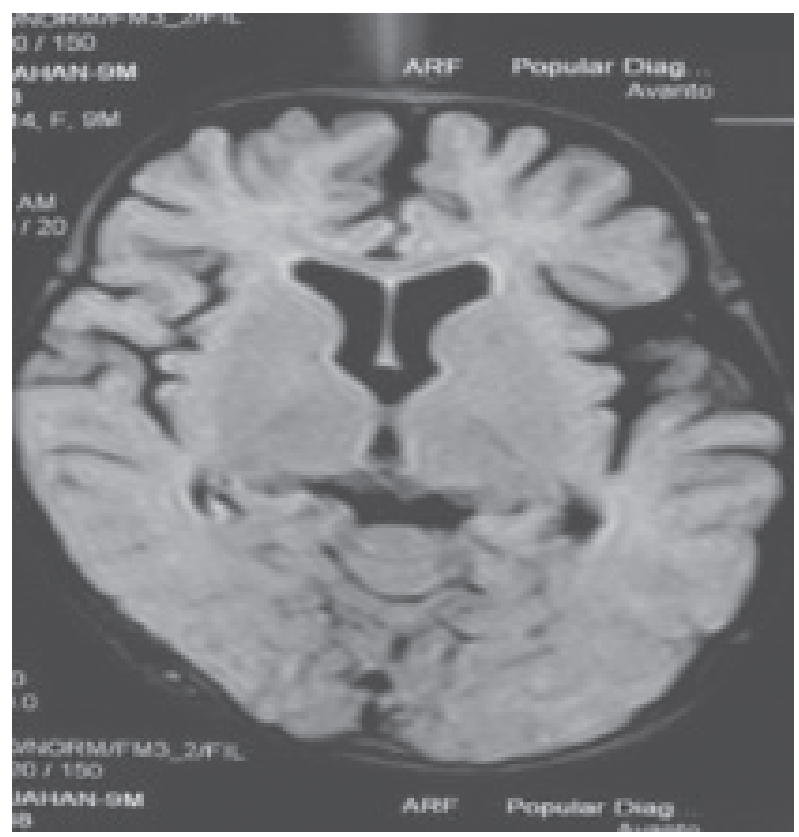

Fig-3: Moderate cerebral atrophy (marked in parietal, frontal and temporal lobe).

\section{Discussion}

Lesch-NyhanSyndrome, is an X-linked recessive metabolic disorder. Over 400 causative HGPRT1 gene mutations have been described ${ }^{18}$. Prevalence has been estimated at between $1 / 235000$ and 1/380000 live-births. ${ }^{19}$ Although LNS syndrome is an X-linked recessive disorder, there have been a few reported cases involving female patients because of point or spontaneous mutation of gene. ${ }^{3}$ The condition is almost exclusively diagnosed in the paediatric population though new diagnosis has been documented in adults. ${ }^{20}$

Benz CMK et $a^{21}$ described the self-destructive behaviour of patients with LNS usually starts between the ages of 1.5 to 2 years or shortly after the primary teeth eruption. The auto-aggressive behaviour is manifested as lips and tongue biting, scratching with the fingernails, head banging. In this case, self destructive behavior like biting of own lips, fingers and tongue started at the age of 7 months.

Visseret.al. support the theory that the neurobehavioral deficits seen in LNS may be related to basal ganglion dysfunction. Further study is required to identify if the neurobehavioral deficits seen in LNS are due to the enzyme deficiency, to the resulting hyperuricaemia, or to some other cause ${ }^{22}$. The neurologic and behavioral abnormalities that are seen in LNS as generalized muscular weakness, athetoid or clonic movements, occasional seizures, positive Babinski's signs, and self-mutilative behaviors. ${ }^{23}$ Neurological and behaviorabnormalities werealso observed in this case, she had hypotonia in lower limbs, repetitive movement of upper left arm and left leg, and seizure.

Brian MS et al. described developmental abnormalities occur and mental retardation is usually severe, IQ values range from 40 to $80 .{ }^{23}$ In this case, She had global developmental delay evidenced by neck control at 8 months of her age, she can sit but can not stand without support and unable to walk till date, she can speak only monosyllables.

HGPRT is a salvage enzyme that plays an essential role in recycling purines. When the HGPRT enzyme is deficient,increased uric acid levels and predispose gout, nephrolithiasis and eventual chronic kidney disease. Renal disease results from intratubular uric acid deposits and interstitial deposits of urate crystals. ${ }^{24}$ Although this case have not had nephrolithiasis but she was having dribbling of urine 
since birth and in our hospitalshe was diagnosed as UTI and treated with IV antibiotics. But after evaluation, MCU report showed grade 2 VUR which is very unlikely in LNS and there is no single case has reported till date so far. So as in LNS, nephrolithiasis is common but should be kept in mind about other kidney disease could occur and thoroughly investigated, as in this case she had grade 2VUR.

The diagnosis of LNS is confirmed by clinical, biochemical, enzymatic, and molecularanalyses. In this case, despite fulfilling the diagnosis criteria of LNS, the patient's serial serum uric acid only showed normal uric acid. Kaufman JM et al. described in young children, renal function is quite efficient in eliminating uric acid into the bladder, and hence, they may have borderline hyperuricemia due to increased renal clearance. ${ }^{25}$

Yamada Y et al. described the HPRT gene is localized to the Xq26 region, and the complete amino acid sequence for HPRT is known to be $44 \mathrm{~kb}$, that converts hypoxanthine intoinosinic acid and guanine into guanylic acid. To date, more than 300 diseaseassociated mutations in the HPRT genehave been identified. ${ }^{26}$ To confirm the diagnosis genetic testing is important, although we have limited facility regarding modern test of genetic analysis so, we have diagnosed LNS clinically.

Currently, there is no cure for the syndrome; prevention is therefore the standard of care. Over the years many inventive and effective approaches involving an assortment of hand restraints, dental mouth guards, and dental extractions have been utilized ${ }^{4,5,11}$ and with appropriate allopurinol treatment, renal function is generally preserved. It has no reported effect on behavioral and neurological symptoms. Allopurinol is efficacious and generally safe as treatment for uric acid overproduction in patients with HPRT deficiency. ${ }^{17}$ This case is also treated with allopurinol along with treatment of UTI (IV antibiotics).

This case is having grade 2 VUR, regarding management of VUR, spontaneous resolution rate is $70-90 \%$ in VUR grade 1-3 and antibiotic prophylaxis is the cornerstone of management; UTI must be recognized promptly and treated aggressively. Regular timed voiding, every $4-5$ hours is very helpful. BP, height, weight and serum creatinine level are recorded every 6 months, MCU is performed every 18-24 months, antibiotic prophylaxis can be discontinued after reflux resolves.
In conclusion, there is no standard method for the prevention of this self-mutilation. Appropriate preventive methods need to be developed for each patient based on reasonable consideration. LNS usually associated with nephrolithiasis, joint pain, and a progressive neurological disorder, resulting in retardation of mental and motor development. But VUR is very uncommon association with LNS and no single case has been reported till date so far and should be kept in mind that VUR could be an association of LNS. If not diagnosed early may lead to kidney damage.

\section{References}

1. Taira T, Kobayashi T, Hori T. Disappearance of self-mutilating behavior in a patient with LeschNyhan syndrome after bilateral chronic stimulation of the globuspallidusinternus. J Neurosurg. 2003;98:414-16.

2. Nyhan, WL. Lesch-Nyhan Disease. J Hist Neurosci 2005;14:1-10.

3. Lee JH, Berkowitz RJ, Choi BJ. Oral selfmutilation in the Lesch-Nyhan syndrome. ASDC J Dent Child. 2002;69:66-69.

4. Evans J, Sirikumara M, Gregory M.Lesch-Nyhan syndrome andthe lower lip guard. Oral Surg Oral Med Oral Pathol. 1993;76:437-40.

5. Smith BM, Cutilli BJ, Fedele M.Lesch-Nyhan syndrome. A case report. Oral Surg Oral Med Oral Pathol. 1994;78:317-18.

6. Steadman RH, McIntosh G, Gross BD.LeschNyhan syndrome. J Oral Maxillofac Surg. 1982; 40:750-52.

7. Cusumano FJ, Penna KJ, Panossian G.Prevention of selfmutilation in patients with Lesch-Nyhan syndrome: review of literature. ASDC J Dent Child. 2001;68:175-78.

8. Shoptaw JT, Reznik JI. Lesch-Nyhan Syndrome: report of three cases in one family. ASDC J Dent Child. 1978;45:403-07.

9. Salman RA, Glicman RS, Super S.Lesch-Nyhan syndrome: report of two cases. J Oral Med. 1987;42:10-13.

10. Gibbs DA, McFadyen IR, Crawfurd MD, De Muinck Keizer EE, Headhouse-Benson CM,Wilson TM.etal.First-trimester diagnosis of Lesch-Nyhan syndrome. Lancet. 1984;2: 1180-83. 
11. Christie R, Bay C, Kaufman JA.Leschnyhan disease: Clinical experience with nineteen patients. Dev Med Child Neurol. 1982;24: 293-306.

12. Jeong TS, Lee JH, Kim S, Kim JH, Tootla RGH. A Preventive Approach to Oral Self-mutilation in Lesch-Nyhan Syndrome: A Case Report. Pediatric Dentistry 2006; 28:4

13. LaBanc J, Epker BN.Lesch-Nyhan syndrome: surgical treatment in a case with lip chewing. $A$ case report. J Maxillofac Surg. 1981;9:64-67.

14. Rinat C, Zoref-Shani E, Ben-Neriah Z, Bromberg Y, Becker-Cohen R, Feinstein S, et al. Molecular, biochemical, and genetic characterization of a female patient with LescheNyhan disease. Mol Genet Metab. 2006;87:249e52.

15. Jinnah HA, Harris JC, Nyhan WL, O'Neill JP. The spectrum of mutations causing HPRT deficiency: an update. Nucleosides Nucleotides Nucleic Acids. 2004;23:1153e60.

16. Yamada Y, Nomura N, Yamada K, Wakamatsu N. Molecular analysis of HPRT deficiencies: an update of the spectrum of Asian mutations with novel mutations. Mol Genet Metab. 2007;90:70e6.

17. Torres RJ, Prior C, Puig JG. Efficacy and safety of allopurinol in patients with hypoxanthineguanine phosphoribosyltransferase deficiency. Metabolism. 2007;56:1179-86.

18. Torres RJ, Puig JG, Jinnah HA. Update on the phenotypic spectrum of Lesch-Nyhan disease and its attenuated variants. Curr Rheumatol Rep. 2010;14:189-94.
19. Torres RJ, Puig JG. Hypoxanthine-guanine phosphoribosyltransferase (HPRT) deficiency: Lesch-Nyhan syndrome. Orphanet J Rare Dis. 2007;2:48.

20. Jinnah HA, Ceballos PI, Torres RJ. Attenuated varaints of Lesch-Nyhan disease. Brain. 2010;133:671-89.

21. Benz CMK, Reeka-Bartschmid AMT, Agostini FG.Case report: the Lesch-Nyhan syndrome. European journal of paediatric dentistry. 2004;2:110-14

22. Visser JE, Bar PR, Jinnah HA. Lesch-Nyhan disease and the basal ganglia. Brain Res Rev. 2000;32:449-75.

23. Brian MS, Bruce JC, Michael F.Lesch-Nyhan syndrome. Oral surc oral med oral path. 1994;78:317-8

24. Doucet BP, Jegatheesan D, Burke J.Late diagnosis of Lesch-Nyhan disease variant BMJ. 2013;10:1-3.

25. Kaufman JM, Greene ML, Seegmiller JE. Urine uric acid to creatininertio e a screening test for inherited disorders of purine metabolism. Phosphoribosyltransferase (PRT) deficiency in X-linked cerebral palsy and in a variant of gout. J Pediatr. 1968;73:583e92.

26. Yamada Y, Nomura N, Yamada K, Wakamatsu N. Molecular analysis of HPRT deficiencies: an update of the spectrum of Asian mutations with novel mutations. Mol Genet Metab. 2007; 90:70e6. 